\title{
A PROPERTY OF THE REAL LINE EQUIVALENT TO THE CONTINUUM HYPOTHESIS
}

\author{
H. H. CORSON
}

1. Introduction. Edwin Hewitt has asked if the real line $R$ is a normal topological space (see below for the definition of this term) when it is given the topology $I$ which is defined in the following way. Fix a Hamel basis $H$ for $R$ over the rational numbers. For $x \in R$ and $a \in H$, let $x_{a}$ denote the $a$ th coordinate of $x$ in its expansion with respect to $H$. Now, for each countable subset $K \subset H$ and each $\epsilon$ such that $0<\epsilon \leqq \infty$, define

$$
V(K, \epsilon)=\left\{x \in R:|x|<\epsilon \text { and } x_{a}=0 \text { for } a \in K\right\} .
$$

Then $J$ is the group topology on $R$ that has the collection of all possible $V(K, \epsilon)$ as a basis of open sets at 0 . The space $(R, \Im)$ is obviously completely regular, since it is a topological group. Theorem 1 answers Hewitt's question. Before stating the theorem, let us recall that a topological space $X$ is normal if any two disjoint closed sets have disjoint neighborhoods.

THEOREM 1. The real line $R$ under the topology $I$ defined above is normal if and only if the continuum hypothesis is true.

2. Proof of sufficiency. First a lemma is stated, next it is pointed out how the lemma implies the desired result, and finally a proof for the lemma is given. For use in stating the lemma, recall that a collection $\mathfrak{u}$ of disjoint subsets of a topological space $X$ is discrete if $x \in(U \mathcal{u})^{-}$implies that $x \in U^{-}$for some $U \in \mathcal{U}$. (The bar indicates closure in $X$.) As for notation, we adopt the convention that $i$ and $j$ run over all integers $\geqq 0$, and $n$ runs over all integers $\geqq 1$.

Lemma 1. If the continuum hypothesis is true, then there exist a countable number of subsets $R_{i}$ of $R$ and a countable number of collections $V_{i}$ such that

(1) $R=U_{i} R_{i}$;

(2) for $x \in R_{i}$, there is a unique $V_{i, x} \in V_{i}$ with $x \in V_{i, x}$;

(3) for $x \in R_{i}, y \in R_{i}, V_{i, x} \cap V_{i, y}=\varnothing$ if $x \neq y$;

(4) for each $i, v_{i}$ is a discrete open collection.

Let us show how Lemma 1 is applied. From $[2,5.32]$ it will be seen that it is sufficient to prove that $(R, J)$ is paracompact. This latter term may be defined as follows. $X$ is paracompact if each open

Received by the editors August 8, 1960 and, in revised form, September 29, 1960. 
cover of $X$ has an open refinement consisting of a countable union of discrete collections. (See $[2,5.28]$.) Now, if $u$ is an open cover for $R$, choose for each $x \in R$ a $U_{x} \in \mathcal{U}$ with $x \in U_{x}$. Then define $W_{i}$ $=\left\{V_{i, x} \cap U_{x}: x \in R_{i}\right\}$. It is easy to verify that $W=U_{i} W_{i}$ has the desired properties.

Proof of Lemma 1. In the way of notation, define for each integer $i \geqq 0$

$$
T_{i}=\left\{x \in R: x_{a} \neq 0 \text { for exactly } i \text { of the } a \in H\right\} .
$$

It will be proved that there are a countable number of subsets $T_{i, n}$ of $T_{i}$ and a countable number of collections $\mathscr{W}_{i, n}$ such that

(1) $T_{i}=\bigcup_{n} T_{i, n}$;

(2) for $x \in T_{i, n}$, there is a unique $W_{n}(x) \in W_{i, n}$ with $x \in W_{n}(x)$;

(3) for $x \in T_{i, n}$ and $y \in T_{i, n}, W_{n}(x) \cap W_{n}(y)=\varnothing$ if $x \neq y$;

(4) for each $n, W_{i, n}$ is a discrete open collection.

(Note that in (4) we mean discrete and open with respect to $(R, J)$.) It is not difficult to verify that Lemma 1 is a consequence of this.

We begin by using the continuum hypothesis to number the Hamel basis $H$ from 1 to (but not including) $\Omega$, where $\Omega$ is the first uncountable ordinal. For $1 \leqq \alpha<\Omega, a(\alpha)$ will denote the $\alpha$ th $a \in H$. Also, for $x \neq 0$, let $\delta_{x}$ be the largest ordinal $\alpha$ such that $x_{a(\alpha)} \neq 0$. Now introduce a relation on $R$ by defining $y<x$ to mean that $x \neq y$, but $y_{a} \neq x_{a}$ only if $x_{a} \neq 0$ and $y_{a}=0$. Note that each $x$ has a finite number of predecessors.

For $x \neq 0$, define

$$
W(x)=\left\{z \in R: z_{a(\alpha)}=x_{a(\alpha)} \text { for } \alpha \leqq \delta_{x}\right\} ;
$$

and for each $n$, define

$$
W_{n}(x)=\{z \in W(x):|z-y|>1 / n \text { for all } y<x\} .
$$

When $i>0$, let $W_{i, n}=\left\{W_{n}(x): x \in T_{i}\right\}$, and set $T_{i, n}=\left[U W_{i, n}\right] \cap T_{i}$. When $i=0$, let $T_{0, n}=\{0\}$ for all $n$, and let $w_{0, n}=\{R\}$.

Let us check that these sets satisfy (1)-(4) above. This is obvious when $i=0$; hence we suppose that $i>0$. Clearly, for $x \in T_{i}$, one can choose $n$ so large that $|x-y|>1 / n$ for all $y<x$. Hence, for this $n$, $x \in W_{n}(x) \in W_{i, n}$, and (1) follows from this. Moreover, if $x$ and $y$ are both in $T_{i}, W(x) \cap W(y) \neq \varnothing$ only if $x=y$; hence, for $x$ and $y$ in $T_{i, n}, W_{n}(x) \cap W_{n}(y) \neq \varnothing$ only if $x=y$. Property (3) is a consequence of this, and (2) follows from this and the definition of $T_{i, n}$.

In proving (4), let us first verify that $W_{i, n}$ is an open collection. Note that each $W(x)$ is open, since $W(x)=x+V(K, \infty)$, where $K=\left\{a(\alpha): \alpha \leqq \delta_{x}\right\}$. Also, since each member of the usual topology for $R$ is in $\mathcal{J}, U=\{z \in R:|z-y|>1 / n$ for all $y<x\}$ is in J. Hence $W_{n}(x)$ $=W(x) \cap U$ is open. 
Finally, to show that $W_{i, n}$ is a discrete collection, suppose $z \in\left(U W_{i, n}\right)^{-}$. Now $z \in T_{p}$ for some integer $p$. If $p \geqq i$, then let $y$ be defined by choosing $y_{a}=z_{a}$ except for the $p-i$ largest $a$ for which $\boldsymbol{z}_{a} \neq 0$ (where "largest" refers to the ordering of $H$ ); for the excepted $a$, let $y_{a}=0$. For this $y, z \in W(y)$ and $y \in T_{i}$; hence $W(y)$ is a neighborhood of $z$ which meets only one member of $w_{i, n}$, namely $W_{n}(y)$.

All that remains is the case $p<i$. It will be proved that, for $z$ and $p$ as above, this case cannot occur. Suppose $z \in T_{p}, p<i$, and let

$$
U=\{y \in W(z):|y-z|<1 / n\} \text {. }
$$

We will prove that $U$ is a neighborhood of $z$ which does not meet $U W_{i, n}$. Suppose to the contrary that $U \cap W_{n}(x) \neq \varnothing$ for some $x \in T_{i}$, and choose a $y \in U \cap W_{n}(x)$. Note that $z<x$, since $U \cap W_{n}(x) \neq \varnothing$ implies that $W(z) \cap W(x) \neq \varnothing$, and the latter implies that $x_{a(\alpha)}=z_{a(\alpha)}$ for all $\alpha \leqq \delta_{z}$. This leads to a contradiction because $y \in W_{n}(x)$ and $z<x$ imply $|y-z|>1 / n$; but $y \in U$ implies $|y-z|<1 / n$. (Note that $U$ is open for the same reason that each $W_{n}(x)$ is open.)

Corollary. Assume the continuum hypothesis. Then every subspace of $(R, J)$ is paracompact, and hence normal. Also, every subset of $(R, J)$ is an $F_{\sigma}$. (An $F_{\sigma}$ is a subset which is the union of a countable number of closed subsets.)

Proof. Note that Lemma 1 remains true if $R$ is replaced by a subspace $A$ of $R$. Hence $A$ is paracompact as above. Also, observe that the properties of $V_{i}$ imply that each subset of $R_{i}$ is closed. Hence $A=\bigcup_{i}\left(R_{i} \cap A\right)$ with each $R_{i} \cap A$ closed in $(R, J)$.

REMARK. The last assertion of the corollary can be derived without the continuum hypothesis by an argument similar to that appearing in the first paragraph of the proof of Lemma 2 in the next section.

3. Proof of necessity. Throughout this section it will be assumed that $\aleph_{1}<2 \aleph_{0}$. We will suppose that $(R, J)$ is normal and argue for a contradiction. This will follow a sequence of lemmas.

Let $i$ be the least integer such that $T_{i}$ is of second category in $R$-where, from here on, $R$ will denote the real numbers under their usual topology.

Lemma 2. $X=\mathrm{U}_{j} T_{i+j}$ is a normal subspace of $(R, \mathfrak{J})$.

Proof. It will be proved that $X$ is an $F_{\sigma}$ in $(R, \mathfrak{J})$. It is sufficient to show this, since it is well known that an $F_{\sigma}$ in a normal space is normal. Define, for integers $j$ and $n, T_{j, n}=\left\{x \in T_{j}:|x-y|>1 / n\right.$ for all $y<x\}$. (The $T_{j, n}$ of the last section could have been defined this way.) One may easily verify that $T_{j}=U_{n} T_{j, n}$. Hence it will suffice to prove that each $T_{j, n}$ is closed in $(R, J)$. 
To accomplish the latter, first suppose that $y \in T_{k} \backslash T_{j, n}(k \geqq j)$ and $K=\left\{a \in H: y_{a} \neq 0\right\}$. Then $y+V(K, \infty)$ is an open neighborhood of $y$ which does not meet $T_{j, n}$. Now assume that $y \in T_{k}(k<j)$ and that $K$ is as above. Then $y+V(K, 1 / n)=V$ does not meet $T_{j, n}$. In fact, if $x \in T_{j} \cap V$ then $y_{b}=x_{b}$ for all $b$ such that $y_{b} \neq 0$. Hence $y<x$, but $|x-y|<1 / n$. It follows that $x \in^{\prime} T_{j, n}$.

For convenience, let us suppose that, whenever a rational number is written in the form $m / n$, we have $m$ and $n$ relatively prime. Define $A$ to be the set of $x \in T_{i}$ such that, for any $a$, the $m$ in the expression $x_{a}=m / n$ is even. Let $B=T_{i} \backslash A$. It follows by an argument similar to part of the proof of Lemma 2 that each subset of $T_{i}$ is closed in $X$. Hence $A$ and $B$ are both closed in $X$. By the normality of $X$, one may choose disjoint $U$ and $V$, each open subsets of $X$, such that $A \subset U$ and $B C V$.

By the definition of the topology $J$, one may choose, for each $x \in \dot{A} \cup B$, a countable $K(x) \subset H$ and $\epsilon(x)>0$ such that $V(x)$ $=[x+V(K(x), \epsilon(x))] \cap X$ is contained in $U$ if $x \in A$, or in $V$ if $x \in B$. Using this notation we will now describe some of the properties of $A$ and $B$.

\section{Lemma 3. $A$ is of second category in $R$.}

Proof. Either $A$ or $B$ is of second category in $R$, since $A \cup B=T_{i}$. For $x \in B, 2^{n} x \in A$ if $n$ is large. Let $B_{n}=\left\{x \in B: 2^{n} x \in A\right\}$. If $B$ is of second category in $R$, then so is $B_{n_{0}}$ for some $n_{0}$. Hence $A$, which contains $2^{n_{0}} B_{n_{0}}$, is of second category in $R$.

For $S \subset R$ and $K \subset H$, let $S(K)=\left\{x \in S: x_{a}=0\right.$ when $\left.a \in K\right\}$.

LEMMA 4. If $S \subset T_{i}$ is of second category in $R$, then $S(K)$ is of second category in $R$ for each countable subset $K$ of $H$.

Proof. Let $g K$ denote the group generated by $K$. Suppose $x \in g K \backslash\{0\}$. Let $S_{x}=(S-x)(K)$. Since $S_{x} \subset\left\{T_{r}: 0 \leqq r<i\right\}, S_{x}$ is of first category in $R$ by the minimality of $i$. Hence $S_{x}+x$ is of first category in $R$. Since $K$ is countable, $T=U\left\{S_{x}+x: x \in g K \backslash\{0\}\right\}$ is of first category in $R$. Consequently, $S(K)=S \backslash T$ is of second category in $R$.

For each set $S$ let $|S|$ denote the cardinality of $S$. Let us say that an interval $I$ (all intervals which occur here will be open) is $\aleph_{2}$-filled with a subset $S$ of $R$ if, for each $K \subset H,|K|<\aleph_{2}$, one has $|S(K) \cap I|$ $\geqq \aleph_{2}$.

LEMMA 5. If I is $\aleph_{2}$-filled with a set $S$ which is the union of a countable collection of $S_{n}$, then for some $n_{0}$ there are arbitrarily small intervals contained in $I$ which are $\aleph_{2}$-filled with $S_{n_{0}}$. 
Proof. Suppose to the contrary that, for each $n$, there is some $\theta(n)>0$ such that, given an interval $J$ of length less than $\theta(n)$, one may choose $K_{J} \subset H$ with the property that $\left|J \cap S_{n}\left(K_{J}\right)\right|<\aleph_{2}$ and $\left|K_{J}\right|<\aleph_{2}$. For a fixed $n$, let $J_{1}, J_{2}, \cdots$ be a countable cover of $I$ by intervals of length less than $\theta(n)$. Define $K_{n}$ as $U_{j} K_{J_{j}}$, and $K$ as $\bigcup_{n} K_{n}$. Then $S_{n}\left(K_{n}\right) \subset \bigcap_{j} S_{n}\left(K_{J_{j}}\right)$; hence $S_{n}\left(K_{n}\right) \cap I \subset \bigcup_{j}\left(S_{n}\left(K_{J_{j}}\right) \cap J_{j}\right)$, and $\left|S_{n}\left(K_{n}\right) \cap I\right|<\aleph_{2}$. Also, $S(K) \subset \bigcup_{n} S_{n}\left(K_{n}\right)$; hence $|S(K) \cap I|$ $\leqq\left|U_{n}\left(S_{n}\left(K_{n}\right) \cap I\right)\right|<\aleph_{2}$. Since $|K|<\aleph_{2}$, this contradicts the assumption that $I$ is $\aleph_{2}$-filled with $S$.

LEMma 6. Each interval $I$ is $\boldsymbol{\aleph}_{2}$-filled with $B$.

Proof. Suppose $|K|<\boldsymbol{\aleph}_{2}$. Then $|B(K)|>\boldsymbol{\aleph}_{1}$ as one may easily verify. Let $x \in R \backslash\{0\}$ be chosen such that each interval containing $x$ also contains $\aleph_{2}$ elements of $B(K)$. Choose a rational number $r$ of the form $m / 2^{n}(n>0)$ such that $r x \in I$. Note that $r B(K) \subset B(K)$, since $m$ and $2^{n}$ are relatively prime. Hence $|B(K) \cap I| \geqq|r B(K) \cap I| \geqq \aleph_{2}$.

For the remainder of this section, let $n$ be an integer, and let $I$ be an interval, such that $n$ and $I$ have the properties of the next lemma. (For the statement of Lemma 7 , recall the definition of $\epsilon(x)$ in the expression of $V(x)$.)

LEMMA 7. There are an integer $n$ and an interval $I$ such that (1) if $A_{n}=\{x \in A: \epsilon(x)>1 / n\}$ then $A_{n} \cap I$ is of second category in $R$, (2) I is $\aleph_{2}$-filled with $B_{n}$ where $B_{n}=\{x \in B: \epsilon(x)>1 / n\}$, and (3) $I$ is of length less than $1 / n$.

Proof. For (1) choose an integer $p$ such that $A_{p}$ is of second category in $R$. It is not difficult to show that, for some interval $J, A_{p} \cap L$ if of second category in $R$ for each subinterval $L$ of $J$. Now $J$ is $\boldsymbol{\aleph}_{2}$-filled with $B$ by Lemma 6 , and by Lemma 5 there is a $q$ such that there are arbitrarily small subintervals of $J$ which are $\boldsymbol{N}_{2}$-filled with $B_{q}$. Let $n=\max (p, q)$. Choose $I$ to be a subinterval of $J$ such that (3) is satisfied for $I$ and (2) is satisfied for $B_{n}$ and $I$. It follows that (1), (2), and (3) are satisfied for $n$ and $I$.

This completes the sequence of lemmas, and we now observe the following facts:

(i) If $x \in\left(A_{n} \cup B_{n}\right) \cap I$, then $V(x) \cap I=W(x) \cap I$, where $W(x)$ $=[x+V(K(x), \infty)] \cap X$. For suppose $y \in W(x) \cap I$; then $|y-x|$ $<1 / n<\epsilon(x)$, and $y \in V(x) \cap I$.

(ii) If $y \in W(x) \cap W\left(x^{\prime}\right)$ for $x \in A_{n} \cap I$ and $x^{\prime} \in B_{n} \cap I$, then there is some $y^{\prime} \in W(x) \cap W\left(x^{\prime}\right) \cap I$. In fact, let $a \in H \backslash\left(K(x) \cup K\left(x^{\prime}\right)\right)$ be chosen such that $y_{a}=0$. Now pick a rational number $r$ such that $y^{\prime}=y+r a \in I$. It follows that $y^{\prime}$ has the desired property. 
(iii) For each $x \in A_{n} \cap I$ and each $x^{\prime} \in B_{n} \cap I, W(x) \cap W\left(x^{\prime}\right)=\varnothing$. In fact, suppose $y \in W(x) \cap W\left(x^{\prime}\right)$ for some choice of $x \in A_{n} \cap I$ and $x^{\prime} \in B_{n} \cap I$. Pick $y^{\prime} \in X$ as in (ii). Then by (i), $y^{\prime} \in V(x) \cap V\left(x^{\prime}\right)$, which contradicts the assumption that $U \cap V=\varnothing$.

This section will be completed by proving that (iii) is false. To accomplish this, choose by induction on $\alpha$ an

$$
x(\alpha) \in\left(I \cap A_{n}\right)(\cup\{K(x(\beta)): \beta<\alpha\})
$$

for $1 \leqq \alpha<\Omega$ such that

$$
(x(\alpha))_{a}=0 \text { if }(x(\beta))_{a} \neq 0 \text { for any } \beta<\alpha .
$$

This is accomplished by using Lemma 4 and the fact that $A_{n} \cap I$ is of second category in $R$. Now pick $x_{0} \in B_{n} \cap I$ such that $\left(x_{0}\right)_{a}=0$ if either $a \in U\{K(x(\alpha)): 1 \leqq \alpha<\Omega\}$ or $(x(\alpha))_{a} \neq 0$ for some $1 \leqq \alpha<\Omega$. This is accomplished by using the fact that $I$ is $\boldsymbol{\aleph}_{2}$-filled with $B_{n}$.

We will contradict (iii) by proving that, for a sufficiently large $\alpha, W\left(x_{0}\right) \cap W(x(\alpha)) \neq \varnothing$. In fact, pick $\alpha$ large enough so that $(x(\alpha))_{a}$ $=0$ if either $a \in K\left(x_{0}\right)$ or $\left(x_{0}\right)_{a} \neq 0$. (This is possible because of condi-

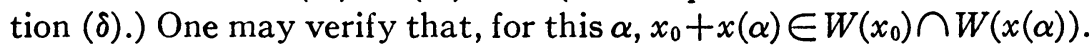
This completes the proof.

4. Remarks. (a) The first three lemmas of $\$ 3$ can be either much simplified or omitted when the Hamel basis is of second category in $R$. However, this is not always so; for instance, a maximal linearly independent subset $H$ of a set which is both of positive measure and of first category in $R$ can be proved to be a Hamel basis. On the other hand, V. L. Klee has pointed out to me that a method due to F. B. Jones [Bull. Amer. Math. Soc. vol. 48 (1942) pp. 115-120] demonstrates the existence of a Hamel basis $H^{\prime}$ which is of second category in $R$.

As in $\S 1$, define a topology $\Im$ on $R$ using $H$, and a topology $J^{\prime}$ using $H^{\prime}$, where $H$ is of first category in $R$ and $H^{\prime}$ is of second category in $R$. One might reasonably conjecture that $(R, J)$ and $\left(R, J^{\prime}\right)$ are homeomorphic. However, let $i$ be the identity function on $R$, and let $f$ be defined by extending linearly a fixed one-to-one correspondence between $H$ and $H^{\prime}$. It can be proved that $f$ is not continuous and that $i$ is not necessarily continuous, where both are considered as functions from $(R, J)$ to $\left(R, J^{\prime}\right)$. Hence, it does not seem that a natural homeomorphism exists, and it is necessary to have a proof that applies to either possibility for $H$.

(b) There are several other topologies for $R$ whose properties complement those of $\mathfrak{J}$. Define $\mathfrak{J}(\boldsymbol{N}, \boldsymbol{\epsilon})$ to be the group topology on $R$ 
generated by all $V(K, \epsilon)$ with $|K|<\boldsymbol{\aleph}$ and $\epsilon>0$, and define $\Im(\boldsymbol{N})$ as the group topology generated by all $V(K, \infty)$ with $|K|<\aleph$. Without any assumptions about the continuum hypothesis one may prove that $J\left(\boldsymbol{\aleph}_{0}, \boldsymbol{\epsilon}\right)$ is not normal, that $J\left(2^{\aleph_{0}}, \epsilon\right)$ is normal (and paracompact), and that $\mathfrak{J}(\boldsymbol{\aleph})$ is normal (and paracompact) for any $\boldsymbol{\aleph}$. Moreover, for $\mathfrak{J}(\boldsymbol{N})$ with $\boldsymbol{N} \leqq \boldsymbol{N}_{1}$, it may be proved that this topology is Lindelöf by the methods of [1, Proposition 3]. (A space is Lindelöf if each open cover has a countable subcover. See [2,5.Y] for a proof that Lindelöf implies paracompact.)

(c) Note that it is also true that $2 \boldsymbol{\aleph}_{0}=\boldsymbol{\aleph}_{1}$ if and only if $(R, J)$ is paracompact; but a proof of this may be constructed which is not so involved as that for Theorem 1 .

\section{REFERENCES}

1. H. H. Corson, Normality in subsets of product spaces, Amer. J. Math. vol. 81 (1959) pp. 785-796.

2. John L. Kelley, General topology, New York, D. Van Nostrand Publishing Company, 1955.

UNIVERSITY OF WASHINGTON 